\title{
SISTEMATIZAÇÃO DAASSISTÊNCIA DE ENFERMAGEM E CLÍNICA AMPLIADA: DESAFIOS PARA O ENSINO DE SAÚDE MENTAL
}

Cláudia Mara Tavares', Lucas Marvilla Mesquita

Objetivo: A Sistematização da Assistência de Enfermagem é uma metodologia para organizar e realizar o cuidado de enfermagem orientado por princípios científicos. A Clínica Ampliada pressupõe uma abordagem singular do sujeito de forma integral, interdisciplinar e compartilhada. Neste artigo fazemos uma reflexão sobre os desafios que essas duas perspectivas trazem para inovar o ensino de saúde mental, procurando identificar interseções e contrapontos teóricos e formas de apropriação pelos enfermeiros de saúde mental. A articulação entre essas duas abordagens amplia a perspectiva de trabalho do enfermeiro em favor do cliente, valorizando suas potencialidades, transformando o processo de cuidar em algo mais dinâmico, participativo e solidário. O principal desafio posto para o ensino de enfermagem em saúde mental é levar o aluno a aprender a agir em cenário de incertezas por meio de práticas profissionais sistematizadas, criativas, autônomo-dialógicas e que levem ao desenvolvimento da profissão considerando o trabalho interdisciplinar.

Descritores: Processo de Enfermagem; Saúde Mental; Ensino em Saúde.

\section{SYSTEMATIZATION OF NURSING AND CLINICALASSISTANCE EXPANDED: CHALLENGES FOR MENTAL HEALTH EDUCATION}

Objective: The systematization of nursing care is a methodology in order to organize and make nursing care oriented by scientific principles. The expanded clinical assistance presupposes a singular personal approach through an integral, interdisciplinary and shared practices. This essay reflects on the challenges which these two perspectives bring to innovate the mental health education, intending to identify theoretical intersections and counterpoints and also its appropriation by mental health nurses. The articulation between these two approaches expand the working perspective of the nurses in favor of the patient, valuing their potentialities, making the care process more dynamic, interactive and supportive. The main challenge of mental health nursing education is to take the students to learn how to act in uncertain scenarios by professional, systematic, creative, autonomous-dialogical practices which also take them to their professional development considering interdisciplinary work.

Descriptors: Nursing Processes; Mental Health; Health Education.

\section{SISTEMATIZACIÓN DE LAASISTENCIA DE ENFERMERÍA Y CLÍNICAAMPLIADA: DESAFÍOS PARA LA ENSEÑANZA DE SALUD MENTAL}

Objetivo: La sistematización de laasistencia de la enfermería es una metodología para organizar y realizar el cuidado de la enfermería orientado por principios científicos. La clínica ampliada presuponeun enfoque singular delsujeto a través de un enfoque integral, interdisciplinar y compartido. En este artículo hacemos una reflexión sobre losdesafíos que estas dos perspectivastraen para innovarlaenseñanza de la salud mental, buscando identificar intersecciones y contrapuntos teóricos y formas de apropiación por losenfermeros de salud mental. La articulación entre estos dos enfoques amplia la perspectiva de trabajo Del enfermeroen favor del cliente, valorizando sus potencialidades, transformando elproceso de cuidar en algo más dinámico, participativo y solidario. El principal desafio puesto para laenseñanza de la enfermería de la salud mental es llevar al alumno a aprender a actuaren un escenario de incertidumbres por medio de prácticasprofesionales sistematizadas, creativas, autónomas - dialógicas y que lleven al desarrollo de laprofesión considerando eltrabajointerdisciplinario.

Descriptores: Procesos de Enfermería; Salud Mental; Educación em Salud.

IUniversidade Federal Fluminense/RJ.

Autor correspondente: Cláudia Mara Tavares. E-mail: claudiamarauff@gmail.com 


\section{INTRODUÇÃO}

Mudanças ocorridas no modelo assistencial em saúde mental e na prática profissional do enfermeiro, engendradas pelo Movimento da Reforma Psiquiátrica, exigeminovaçõesequivalentes no processo de formação. Em decorrência dessas mudanças, o leque de ações de enfermagem foi ampliado e ao mesmo tempo posto em interação e interdependência com demais profissionais, visando à integralidade dos cuidados em saúde. Nesse contexto, propõe-se um ambiente de trabalho de intensificação de trocas e afetos entre diferentes atores sociais, desconstrução de práticas de objetivação da doença mental e (re) construção de práticas que consideram a alteridade do sujeito.

O atual modelo assistencial de atenção à saúde mental preconiza a substituição progressiva dos hospitais psiquiátricos por dispositivos extra-hospitalares inseridos no território em que vivem os usuários, trazendo maior complexidade na abordagem aos mesmos e suporte às suas famílias. A lógica de trabalho proposta nos serviços é bastante diversa daquela tradicionalmente engendrada na formação em saúde -focada na formação de especialistas, centrado na doença, com sua busca pela verdade no interior do sujeito, preocupado com o manejo de técnicas e tecnologias.

A reorganização das práticas de saúde mental no contexto de consolidação do Sistema Único de Saúde (SUS) está diretamente relacionada às atividades desenvolvidas na formação dos profissionais de saúde. Contudo, as instituições de ensino não têm como tradição o diálogo e o trabalho conjunto com os serviços de saúde. A elaboração de projetos que articulem teoria e prática, numa perspectiva ampla do cuidar, favorecendo a formação de profisısionais compromissados com a sociedade e seus problemas de saúde, é um dos desafios que precisam ser enfrentados ${ }^{(1)}$.

Estudos avaliativos mais recentes sobre o ensino mostram que as disciplinas curriculares de saúde mental e psiquiatria são marcadas pelo enfoque do que seja normal e patológico no transcorrer do ciclo vital, centradas na psicopatologia e na instituição psiquiátrica. Ainda que haja algumas iniciativas dos docentes de saúde mental na busca por abordagens pedagógicas progressistas, que propiciem a formação de sujeitos críticos e reflexivos, capacitando-os para intervirem em contextos complexos ${ }^{(2.3)}$.

Espera-se que o enfermeiro use em sua prática profissional um método de trabalho para planejar, executar e avaliar suas ações em sincronia com o sistema de saúde. Contudo, existem contradições, resistências e desafios a serem superados em sua práxis. Não há consenso nacional sobre que modelo utilizar, nem garantia da estrutura e recursos necessários para o seu desenvolvimento. Há tensões e conflitos entre modelos de orientação profissional e de assistência multiprofissional em saúde, profissionais da equipe de saúde, instituição de ensino e assistência. Há uma recomendação do Conselho Federal de Enfermagem (COFEN) para o uso da Sistematização da Assistência de Enfermagem (SAE) nos serviços onde haja enfermeiros. Já o Ministério da Saúde, propõe a Clínica Ampliada (CA).

Esse artigo tem como objetivo refletir sobre os desafio sengendrados pela proposta da SAE e da CA para o ensino de enfermagem em saúde mental.

\section{METODOLOGIA}

Trata-se de uma reflexão teórica sobre os desafios que duas perspectivas sobre a prática de cuidar - SAE e CA trazem para inovar o ensino de saúde mental, procurando identificar interseções e contrapontos teóricos e formas de apropriação pelos enfermeiros de saúde mental. Realizou-se com base na literatura disponivel uma formulação discursiva aprofundada, enfocando o tema em estudo e seus desafios para o ensino de enfermagem, estabelecendo analogias e analisando diferentes pontos de vista teóricos e práticos sobre o assunto.

\section{A SISTEMATIZAÇÃO DAASSISTÊNCIA DE ENFERMAGEM EM SAÚDE MENTAL}

A SAE é tida como um instrumento metodológico que orienta e documenta o cuidado profissional de Enfermagem, evidenciando sua contribuição na atenção à saúde da população, aumentando a visibilidade e o reconhecimento profissional do enfermeiro. Operacionaliza-se por meio do Processo de Enfermagem (PE), organizado em cinco etapas inter-relacionadas, interdependentes e recorrentes: histórico de enfermagem, diagnóstico de enfermagem, planejamento, implementação e avaliação de enfermagem ${ }^{(4)}$.

Nos serviços de saúde mental a SAE não é um método usual de trabalho adotado pelos enfermeiros, embora haja algumas experiências bem-sucedidas com sua aplicação. É o caso daexperiência relatada por Toledo(5), que utilizou um instrumento para SAE numa unidade de internação psiquiátrica. Ele verificou que a SAE permitiu à enfermeira a tomada de decisões, oferecendo subsídios para o estabelecimento dos diagnósticos, resultados esperados e ações de enfermagem que sustentam a avaliação do cuidado integral. A SAE constituiu uma alternativa para estabelecer um plano de cuidados coerente com a realidade vivenciada junto ao paciente, fortalecendo a participação do enfermeiro na constituição de projetos terapêuticos no âmbito da equipe multiprofissional.

A SAE vem sendo uma ferramenta útil e importante na tentativa de construir uma prática de enfermagem mais eficaz 
no contexto da reabilitação psicossocial. Nesse cenário, a Consulta de Enfermagem (CE) é tida como uma prerrogativa a ser executada no processo de trabalho do enfermeiro, um meio de acompanhamento do cuidado junto ao usuário, integrado ao Projeto Terapêutico Singular (PTS)(6).

Há escassez de literatura sobre a aplicação, avaliação e eficácia do PE em saúde mental. Os estudos tratam apenas sobre parte do processo de enfermagem. As etapas de histórico e planejamento dos cuidados de enfermagem são as mais executadas, e tidas como contribuição do enfermeiro para a construção do PTS e sua inserção na equipe multiprofissional. Estudos cuja preocupação é construir diagnósticos de enfermagem com a finalidade de criar padrões de cuidado tendem a observar o modelo biológico, estando influenciado pela concepção da prática baseada em evidência e não pela perspectiva relacional, sendo referida a usuários com problemas de saúde cuja reprodução e padronização são factíveis.

Os estudos que versam sobre a prática do enfermeiro em saúde mental pautam-se na descrição de experiências dos enfermeiros sobre o uso da relação terapêutica como foco das intervenções, baseadas na perspectiva de Peplau. Aqueles que propuseram estabelecer diagnósticos de enfermagem em saúde mental, em sua maioria, utilizaram a taxonomia $\operatorname{NANDA}^{(7)}$

O entendimento presente no campo da Saúde Mental (SM) que o cuidado é singular e que seu foco é a relação terapêutica, opõe-se as proposições da prática de enfermagem baseada em evidências, o que pode explicar a resistência dos enfermeiros para adoção da SAE em SM.

A insuficiência de recursos humanos, excesso de atribuições do enfermeiro, desconhecimento da metodologia de trabalho, escassez de recursos materiais, a resistência ao novo, métodos diferentes de registro dificultam a realização do PE em todas as áreas, além de que o enfermeiro, muitas vezes, deixa de cumprir suas atribuições para dar apoio a outros profissionais ${ }^{(8)}$

A visão do cuidado de enfermagem como um fim em si mesmo, com pouca interdependência aos saberes dos usuários e dos demais profissionais da saúde tem levado essa abordagem a ser alvo de muitas críticas, posto que no cenário contemporâneo da saúde, valorizam-se processos participativos de cuidar.

\section{ENFERMAGEM E CLÍNICAAMPLIADA EM SAÚDE MENTAL}

A CA propõe rever o cuidado fragmentado por especialidades, ampliando o agir dos diferentes profissionais de saúde com vista à produção do cuidado integral com centralidade no sujeito. É baseada no conceito de saúde integral e compreende a qualidade de vida como o resultado de fatores biopsicossociais. Seus princípios foram concebidos por Campos ${ }^{(9)}$ como crítica a clínica tradicional centrada na doença e no ato médico. Nega a predominância da determinação social, biológica ou subjetiva dos processos saúde-doença. Considera que todo profissional de saúde que atenda ou cuide de pessoas, e não apenas o médico, realiza clínica, precisando ter plasticidade suficiente para dar conta da variabilidade biológica, psíquica e social dos casos e da imprevisibilidade da vida.

São proposições fundamentais da CA: o acolhimento com destaque para o poder terapêutico da escuta ampliada ao Sujeito; o trabalho interdisciplinar em saúde na perspectiva do cuidado integral e a participação e autonomia do Sujeito na definição do projeto terapêutico ${ }^{(9,10)}$

A CA enquanto diretriz da Política Nacional de Humanização opera por meio de dois dispositivos de gestão da atenção: as Equipes de Referência (ER) e os Projetos Terapêuticos Singulares (PTS) ${ }^{(11)}$. A ER contribui para tentar resolver ou minimizar a falta de definição de responsabilidades, de vínculo terapêutico e de integralidade na atenção à saúde, têm a responsabilidade pela condução de um caso individual, familiar ou comunitário(10). O PTS é um conjunto de propostas de condutas terapêuticas articuladas, para um sujeito individual ou coletivo, que incorpora a contribuição de várias especialidades e de distintas profissões ${ }^{(12)}$

A participação da enfermagem na CA é um projeto em construção. Compreendida como prática social, a enfermagem vem atendendo as necessidades sociais da população a partir de determinantes históricos e políticos. Como profissão inserida no SUS e representando uma força de trabalho equivalente há cerca de $50 \%$, não pode estar alheia às diretrizes de atenção à saúde contemporânea.

A prática clínica engendrada por enfermeiros na atenção básica na perspectiva da CA vem se constituindo no interior da prática médica hegemônica, mediada por tensões e conflitos. Nesse contexto, os enfermeiros consideram que desenvolvem uma espécie de "pseudoclínica", já que persistem na maioria das unidades, a centralidade na atividade médica e condições estruturais e organizacionais desfavoráveis. Algumas atividades de enfermagem são consideradas pelos enfermeiros como integrantes da CA pelo grande investimento que fazem em acolhimento nas consultas de enfermagem, visita/atendimento domiciliar, trabalho em grupo, orientação a auxiliares de enfermagem e agentes comunitários de saúde e o apoio ao atendimento do médico ${ }^{(13)}$.

A enfermagem na atenção em saúde mental resguarda a noção de cuidado como foco da sua ação, ressaltando a intersetorialidade como um dos atuais dispositivos para a ampliação e o fortalecimento das ações no campo da saúde mental, asseverando que a saúde, sozinha, não dá conta 
da complexidade do cuidar, carecendo de outros saberes. O desenvolvimento de intervenções de enfermagem em saúde mental é construído no cotidiano dos encontros entre profissionais e usuários, em que ambos criam novas ferramentas e estratégias para compartilhar e construir juntos o cuidado em saúde. A perspectiva da integralidade em saúde mostra-se a mais adequada para este campo, sendo o Matriciamento em Rede um dispositivo potente para resolução de problemas ${ }^{(14)}$.

Nos serviços substitutivos as enfermeiras apresentam discursos e concepções de saúde que corroboram para a desconstrução do saber psiquiátrico e para a superação das práticas manicomiais, demonstrando compromisso com o desenvolvimento de suas ações profissionais a partir do trabalho interdisciplinar.

Matos $^{(15)}$ destaca que para atuar no Centro de Atenção Psicossocial (CAPS), principal dispositivo de atenção em SM, é indispensável extrapolar as ações e os saberes convencionais de enfermagem, interligando ações próprias a outros campos de saberes e profissões. No CAPES a enfermeira articula casos clínicos, em contato com o território e com outros setores sociais. Quando na gestão da clínica ampliada, desenvolve a função de referência dos casos, constrói e consolida projetos terapêuticos. É sobre as ações nucleares de cuidado direto a pessoa com transtorno psiquiátrico que a enfermagem se aproxima melhor dos princípios da clínica ampliada, havendo, entretanto, maior dificuldade no desenvolvimento das ações gerenciais de enfermagem, ainda arraigadas no modelo tradicional centralizador.

Pelo exposto não há dúvida de que os princípios da clínica ampliada são desejáveis e oportunos para a qualificação da assistência de enfermagem em saúde mental, mas por que eles não se efetivam na prática de enfermagem contemporânea? Vislumbram-se como possiveis explicações: a força do paradigma tradicional ainda dominante nos serviços de saúde; a hegemonia do saber médico; a falta de direcionamento dos gestores de enfermagem para adoção de um modelo assistencial específico; a falta de estrutura e de políticas de saúde adequada; a falta de regulação/monitoramento e avaliação pública dos serviços de saúde; formação inadequada/insuficiente dos profissionais de enfermagem e ausência de processos de educação permanente.

\section{DESAFIOS PARA INOVAÇÃO DO ENSINO DE ENFERMAGEM EM SAÚDE MENTAL}

As Diretrizes Curriculares Nacionais do Curso de Graduação em Enfermagem (DCN) ${ }^{(16)}$ orientam a formação do enfermeiro para a integralidade do cuidado, o que exige desenvolver práticas de ensino que favoreçam a compreensão e desenvolvimento da integralidade na formação acadêmica e profissional.

O ensino de Enfermagem Psiquiátrica/SaúdeMental, além de seguir as DCN, deve orientar-se por princípios da Reforma Psiquiátrica brasileira, buscando principalmente inserir os estudantes nos espaços abertos de atenção à pessoa em sofrimento psíquico e não os manter exclusivamente no âmbito hospitalar ${ }^{(2)}$

Ressalta-se que o modo tradicional como se constituem as práticas de formação e de cuidar ainda não foi superado. Convive-se com a assistência manicomial ao lado dos novos dispositivos assistências em saúde mental. O Brasil tem atualmente cerca de 170 hospitais psiquiátricos ${ }^{(17)}$. A despeito das críticas, o hospital psiquiátrico, continua sendo um importante órgão empregador de enfermeiros e campo de sua formação clínica.

Os reflexos da formação dos enfermeiros em espaços manicomiais são sentidos nos demais serviços que integram a rede de atenção em saúde mental. Nos ambulatórios, por exemplo, os enfermeiros ainda encontram resistências e limitações para integrar efetivamente as equipes de saúde mental, reconhecendo que parte desse problema decorre de suas dificuldades para avaliar aspectos psíquicos dos usuários devido à fragilidade de sua formação para a leitura das relações ${ }^{(7)}$

A mudança na formação ou o investimento em projetos de educação permanente das equipes são medidas frequentemente apontadas para superar o estágio atual de baixa qualidade da prática de enfermagem em SM ou de seu exercício em bases limitadas nos dispositivos assistenciais de saúde mental. Constatam-se nos serviços de saúde mental dificuldades para o uso da SAE, da CA ou de qualquer outra metodologia de atuação profissional de enfermagem fora dos moldes tradicionais ${ }^{(2,3,6,18)}$

A formação em enfermagem depende da qualidade das aprendizagens em contexto clínico, mas os profissionais que se encontram nos serviços de saúde mental precisam de formação/capacitação condizentes às exigências de qualidade assistenciais contemporâneas. Nesse sentido, as instituições de ensino e serviço possuem uma interdependência natural, mas por razões históricas e culturais não atuam de forma sinérgica em prol do fortalecimento do SUS.

Ampliar a visão dos alunos para além do modelo biológico médico-centrado do processo saúde-doença, visando à compreensão do sujeito integrado com o seu meio social, político, econômico e cultural é uma forma de enfrentar a fragmentação da assistência e o trabalho baseado no "modelo funcional"(19)

A interdisciplinaridade é tida como questão central para ultrapassar o aprendizado por meio do saber 
instrumental. Ela oportuniza aos discentes de enfermagem compreender a clínica ampliada como dispositivo imprescindivel para despertar reflexões e operacionalizar mudanças, capacitando-os para explorar relações entre as disciplinas e, com base nelas, solucionar problemas que ultrapas $\neg$ sassem os limites pedagógicos do conteúdo, fortalecendo o movimento entre a ação-reflexão-ação e prática-teoria-prática ${ }^{(20)}$

O ensino crítico, integrado, ativo e criativo é apontado como um meio para enfrentar os problemas vivenciados nos serviços de saúde mental, mas insuficiente para superar os desafios impostos à prática de enfermagem. Condições estruturais, políticas, legais, administrativas, culturais e éticas interferem substancialmente na melhoria da atenção à saúde mental (6,13 15,19)

Vislumbra-se a formação de enfermeiros com maior integração entre as disciplinas e os conhecimentos experimentados em equipe interdisciplinar, colaborando para a formação de profissionais com habilidades e competências que thes permitam encontrar soluções compartilhadas para problemas complexos ${ }^{(15)}$

Salienta-se que os projetos de capacitação devam ser estruturados a partir da problematização do processo de trabalho, objetivando a transformação das práticas profissionais e a organização do trabalho, tendo em vista as necessidades de saúde da população(17)

Assim, para inovar o ensino de saúde mental com base nas necessidades do serviço de saúde mental, é preciso considerar os seguintes desafios: desenvolver experiências mediadas pela perspectiva da interdisciplinaridade, que tem função de alcançar o desenvolvimento de um pensamento complexo nas questões relativas à saúde mental; solidarizar processos de educação permanente; reorientar o currículo de enfermagem com base na perspectiva da clínica ampliada; intensificar o debate sobre os modelos assistenciais em saúde; adotar metodologias de ensino que propiciem maior autonomia do estudante; diversificar experiências de formação para que o estudante de enfermagem amplie competências sociais e comunicacionais; promover currículos integrados, contemplando integração de disciplinas e áreas de conhecimento; aprofundar as bases teóricas que permitam analisar com profundidade as relações terapêuticas com vista o fortalecimento do saber de enfermagem para tomada de decisão em equipe; promover o autoconhecimento do estudante e a consciência do cuidado de si.

\section{Limitações do estudo}

É escassa a literatura disponível sobre o trabalho dos enfermeiros em saúde mental, tanto na perspectiva da SAE quanto da CA o que traz limite para possíveis generalizações do estudo.

\section{Contribuições para a prática}

A principal contribuição do estudo é colaborar para a articulação entre a perspectiva da SAE e da Clínica Ampliada no campo da atenção à saúde mental trazendo uma nova perspectiva para o trabalho do enfermeiro. $O$ estudo permite evidenciar que o tema estudado foi pouco explorado demonstrando a necessidade de se avançar na realização de estudos empíricos.

\section{CONCLUSÕES}

Tendo em vista que a reforma psiquiátrica proporcionou mudanças voltadas para a inserção da pessoa com transtorno psiquiátrico na comunidade, no ambiente familiar e na sociedade, é preciso ultrapassar limites e desafios existentes na prática profissional e na formação acadêmica para fortalecer a profissão enfermagem e a qualidade dos cuidados em saúde mental.

As pesquisas divulgadas evidenciam práticas profissionais restritas, pouco referenciadas e metodologicamente indefinidas. Não há consenso sobre modelos orientadores da prática de enfermagem e os princípios preconizados para atenção em saúde mental são pouco regulados ou avaliados. Boas práticas de enfermagem e experiências inovadoras existem, mas não foram divulgadas o bastante em periódicos científicos, fato que pode ser explicado pelo afastamento dos enfermeiros da academia ou pelo restrito desenvolvimento de pesquisas no âmbito dos serviços.

Os princípios da CA associados a SAE podem fazer avançar a Enfermagem, contribuindo para a construção do cuidado integral. O PE desenvolvido a partir da relação enfermeiropaciente pode ser um avanço na construção de laços para um cuidado de maior visibilidade ao saber e fazer da enfermagem. O desafio persistente é a interdisciplinaridade, sendo preciso articular o saber-fazer da enfermagem na direção de um agir ampliado com os demais profissionais de saúde e os usuários em prol da produção de saúde.

Assim, compreendemos, que o principal desafio contemporâneo para o ensino de enfermagem em saúde mental é motivar o aluno para aprender a agir em cenário de incertezas, por meio de práticas profissionais sistematizadas, criativas, autônomo-dialógicas e que levem ao desenvolvimento da profissão considerando o trabalho interdisciplinar e a complexidade do processo de adoecer humano.

Algumas experiências exitosas no campo da integração ensino-serviço indicam caminhos para a política de educação permanente, formação e ensino na 
graduação em enfermagem em saúde mental, entre os quais destacamos: incorporação do conceito ampliado de saúde para desenvolver o ensino-aprendizagem; estabelecimento de parcerias com serviços e demais profissionais de saúde visando ampliar as oportunidades para práticas interdisciplinares; desenvolvimento das habilidades relacionais e socioemocionais do estudante; e uso de metodologias ativas no processo ensinoaprendizagem.
Alerta-se para a necessidade de consolidação, sustentabilidade e divulgação de experiências inovadoras no ensino de enfermagem em saúde mental, para fazer avançar a ciência de enfermagem com compromisso social.

\section{Contribuição dos autores}

Concepção e/ou desenho: Cláudia Tavares e Lucas Mesquita: análise e interpretação dos dados, redação do artigo, revisão final e revisão crítica: Cláudia Tavares e Lucas Mesquita.

\section{REFERÊNCIAS}

1.Ceccim RB, Feuerwerker LCM. O quadrilátero da formação para a área da saúde: ensino, gestão, atenção e controle social. Physis [Internet]. 2004 June [cited 2019 June 13] : 14(1): 41-65. Availablefrom: http://www.scielo.br/scielo.php?script=sci_arttext\&pi$d=$ S0103-73312004000100004\&lng=en

2. Souza MCBM. O Ensino de Enfermagem Psiquiátrica /Saúde Mental: avanços, limites e desafios. SMAD, Revista Electrónica enSalud Mental, Alcohol y Drogas [Internet]. 2016 [cited 2019 June 2]: 12(3):139146. Available from: http://dx.doi.org/10.5935/1414- 8145.20160040.

3.Skupien SV, Pinto AC, Floriano LSM. Avaliação do ensino de psiquiatria e de saúde mental na graduação de enfermagem: revisão de literatura, 2017.Anais do IV Seminário Internacional de Representações sociais, subjetividade e educação, Curitiba, Paraná.

4. Conselho Federal de Enfermagem. Resolução No 358 de 15 de outubro de 2009. Dispõe sobre a Sistematização da Assistência de Enfermagem e a implementação do Processo de Enfermagem em ambientes, públicos ou privados, em que ocorre o cuidado profissional de Enfermagem e dá outras providências. Brasilia, DF: Conselho Federal de Enfermagem, 2009.Avaiblefrom:http://www.cofen.gov.br/resoluo-cofen-3582009_4384.html

5.Pellegrino TV, Nakamura MS, Rigon FGAP. Sistematização da assistência de enfermagem em unidade de internação psiquiátrica. Revista Baiana de Enfermagem [Internet], 2015[cited 2019 June 13]:29(2):172179. Available from: https://portalseer.ufba.br/index.php/enfermagem/article/view/11707/pdf_130

6.Bolsoni E, Heusy I, Silva Z, Rodrigues J, Peres G, Morais R. Consulta de enfermagem em saúde mental: revisão Integrativa. SMAD [Internet]. 2016 [cited 2019 june 14]:12(4):249-255. Available from: http:// www.revistas.usp.br/smad/article/view/122268

7.Garcia A. Paula RF, Freitas MIP, Lamas JLT, Toledo VP. Processo de enfermagem na saúde mental: revisão integrativa da literatura. Rev. Bras. Enferm. [Internet]. 2017 [cited 2019 Jun 14] ; 70(1): 220-230. Available from: http://www.scielo.br/scielo.php?script=sci_arttextEpid=S0034-71672017000100220\&lng=pt

8. Santos MG et al. Etapas do processo de enfermagem: uma revisão narrativa. Enferm Foco, [Internet].2018 [cited 2019 jul 15]; 8 (2): 49-56. Available from: http://revista.cofen.gov.br/index.php/enfermagem/ article/view/1032/416

9.Campos GWS. Saúde Paidéia. São Paulo: Hucitec, 2003.

10.Campos GWS, Domitti AC. Apoio matricial e equipe de referência: uma metodologia para gestão do trabalho interdisciplinar em saúde. Cad. Saúde Pública [Internet]. 2007 [cited 2019 Jun 14]: 23(2): 399407. Available from: http://www.scielo.br/scielo.php?script=sci_arttext\&pid=S0102-311X2007000200016\&lng=pt

11.Ministério da Saúde (BR). Secretaria Executiva, Núcleo Técnico da Politica Nacional de Humanização. HumanizaSUS: clinica ampliada e

compartilhada [Internet]. Brasilia (DF). 2009.Available from:http:// bvsms.saude.gov.br/ bvs/publicacoes/clinica_ampliada_compartithada. pdf.

12. Ministério da Saúde (BR). Secretaria de Atenção à Saúde. Núcleo Técnico da Política Nacional de Humanização. Clínica ampliada, equipe de referência e projeto terapêutico singular. 2. a edição. Brasilia: Série B. Textos Básicos de Saúde,2007.

13. Matumoto S, Fortuna CM, KawataLS, Mishima SM, Pereira MJB. A prática clínica do enfermeiro na atenção básica: um processo em construção. Rev. Latino-Am. Enfermagem [Internet]. 2011 [cited 2019 June 13] : 19( 1 ): 123-130. Availablefrom: http://www.scielo.br/ scielo.php?script=sci_arttext\&pid=S0104=11692011000100017-\&lngen. http://dx.doi.org/10.1590/S0104-11692011000100017.

14.Alves S.Os sentidos sobre cuidado na atenção à saúde mental no contexto da enfermagem.Dissertação de mestrado, Pontifícia Universidade Católica de Goiás, Goiânia, Programa de Pós-Graduação Stricto Sensu em Psicologia, 2015.

15. Mattos NFMS.O processo de trabalho da enfermagem no CAPS III de Campinas a partir da clínica ampliada e compartilhada. Dissertação (mestrado profissional) - Universidade Estadual de Campinas Faculdade de Ciências Médicas, 2016.

16. Ministério da Educação. (BR). Conselho Nacional de Educação. Câmara de Educação Superior. Resolução CNE/CES n. 3, de 7 novembro de 2001. Institui as diretrizes curriculares nacionais do curso de graduação em Enfermagem. Diário Oficial da República Federativa do Brasil. Brasilia (DF), 9 de novembro de 2001. Seção 1, p. 37.

17. Castro M. Com reforma, número de leitos psiquiátricos no SUS diminuiu 40\%. Barbacena, 22/08/2015. TV Brasil. http://agenciabrasil. ebc.com.br/direitos-humanos/noticia/2015-08/com-reforma-numero-de-leitos-psiquiatricos-no-sus-diminuiu-40-em

18. Tavares CMM, Gama LN, Souza MMT e, Paiva LM, Silveira PG, Mattos MMGR. Competências especificas do enfermeiro de saúde mental enfatizadas no ensino de graduação em enfermagem. Revista Portuguesa de Enfermagem de Saúde Mental [Internet]. 2016 [citado 2019 Jun 13] : ( spe4 ): 25-32. Available from: http://www.scielo.mec.pt/scielo. php?script=sci_arttextEpid=S1647-216020160004000048lng=pt. http://dx.doi.org/10.19131/rpesm.0137.

19.Teixeira GV, OliveiraNR. Considerações sobre a clínica ampliada no processo de enfermagem. Saúde Coletiva [Internet]. 2010 [cited 2019 Mai 13]; 7(38):61-66. Available from: http://www.redalyc.org/articulo.oa?id=84212375006

20.Feitosa RMM, Lima DWC, Almeida ANS, Silveira LC. Pensar e fazer na enfermagem: a interdisciplinaridade na perspectiva da clinica ampliada. Revista Baiana de Enfermagem. 2011[cited 2019 Mai 13]; 25(1): 75-88.Available from: https://portalseer.ufba.br/index.php/enfermagem/article/view/4718/4331

RECEBIDO EM: 18/06/2019

ACEITO EM: 06/09/2019 